(2) Open Access Full Text Article

ORIGINALRESEARCH

\title{
Downregulation of Chemokine CCL20 Involved in Myeloma Cells Resistant to Elotuzumab and Lenalidomide
}

\section{Huihan Wang \\ Hua Shi \\ Xiaowei He \\ Aijun Liao}

Haematology Department of Shengjing Hospital, China Medical University, Shenyang, People's Republic of China
Correspondence: Aijun Liao Haematology Department of Shengjing Hospital, China Medical University, No. 36 Sanhao Street, Heping District, Shenyang, I I0004, Liaoning, People's Republic of China Tel +86-18940259833

Email 1391860764@qq.com
Objective: Few studies have focused on investigating resistance mechanisms in myeloma immunotherapy. This study aimed to explore the relevant factor involved in the resistance of Elotuzumab and lenalidomide.

Methods: Cell models which are resistant to Elotuzumab and lenalidomide were constructed; different expression genes in U266/WT (UW) and resistant UR, UE, and URE cells were detected by using gene expression microarray. RT-qPCR validated CCL20 mRNA expression of four cell lines and patient samples; bioinformatics analysis of CCL20 expressions in NDMM and RRMM; ELISA detected the presence of CCL20 in the plasma of MM patients; constructed UR mouse xenograft model to explore whether or not CCL20 reverse lenalidomide treatment in vivo.

Results: Cell models which are resistant to Elotuzumab and lenalidomide (UR, UE, URE) were successfully constructed. CCL20 gene expression decreased in resistant myeloma cell lines and RRMM patients. Furthermore, RRMM patients were found to have lower levels of CCL20 protein in their plasma compared to NDMM. CCL20 increase the sensitivity of drugresistant myeloma cells to immunomodulatory drugs both in vivo and in vitro.

Conclusion: The expression of CCL20 was decreased in lenalidomide and Elotuzumab resistant U266 cells and in RRMM patients. CCL20 could therefore possibly increase the sensitivity of lenalidomide and Elotuzumab.

Keywords: CCL20, elotuzumab, lenalidomide, drug resistance, multiple myeloma

\section{Introduction}

Multiple myeloma (MM) is a second hematologic malignancy commonly associated with the elderly population. Although the development of new drugs and treatment procedures have resulted in significant improvements in the response and survival rates of $\mathrm{MM}$ patients, it is still incurable, ${ }^{1}$ with many patients still progressing to relapse/refractory MM (RRMM). Immunotherapy has attracted increasing attention in the treatment of multiple myeloma due to its advantages of targeting ability and low toxicity level. ${ }^{2}$ While there are few studies on the mechanism of immunotherapy resistance, the ability of lenalidomide to regulate the immune function as a second generation of immunomodulators has widely been adopted in the treatment of MM. Elotuzumab (Elo) is the second monoclonal antibody drug approved for the treatment of RRMM in combination with lenalidomide and dexamethasone (E-Rd regimen)., ${ }^{3,4}$ The therapeutic target of Elotuzumab is a member of the signal lymphocyte activating molecule family 7 (slamf7, also known as CD319, CS1). CS1 is a cell surface 
glycoprotein molecule, with a very high expression on the surface of myeloma cells and differentiation stages of normal B cells. ${ }^{5}$ Elotuzumab can directly activate NK cells and induce CD16 mediated cytotoxicity to kill myeloma cells with high expressions of CS1. While the E-Rd regimen achieved satisfactory results, there was an emergence of resistance to the drug in some patients. The resistance mechanism of immunotherapy is quite different from that of small molecule chemical drugs. The effectiveness of immunotherapy depends on the normal function of immune cells, especially $\mathrm{T}$ cell function, including recognition, chemotaxis, and the regulation of killing by cytokines. In this study, we developed resistant cell lines with resistance to Elotuzumab and lenalidomide, and we explored the mechanism involved in the resistance to monoclonal antibody and immunotherapy with the primary aim of providing new targets for immunotherapy and uncovering new procedures to reverse drug resistance.

\section{Methods}

\section{Myeloma Cell Culture}

Bone marrow samples of MM patients were sorted using CD138 magnetic beads obtained from Shengjing Hospital of China Medical University. The Medical Ethics Committee at the Shengjing Hospital of China Medical University approved the present study based on the principles of the Declaration of Helsinki. Informed consent was granted by all participating patients in the study, as shown by their endorsement of the consent form.

Myeloma drug-naive U266 cell line (U266/WT, UW) and lenalidomide-resistant cell line (U266/R10R, UR) were provided by Professor Orlowski Robert from the MD Anderson Cancer Centre. UW and UR cell lines were initially exposed to Elotuzumab at $10 \%$ minimum inhibitory concentration (IC10), and cocultured with human peripheral blood mononuclear cells (PBMC) provided by health donor for 48 hours. Subsequently, the surviving cells were collected and sorted with CD138 antibody using flow cytometry. The drug simulation process was replicated until the cell lines were stable with Elo single resistance (U266/ EloR, UE) and double resistance (U266/R10R/EloR, URE). The use of the cell lines was approved by the Medical Ethics Committee at the Shengjing Hospital of China Medical University.

\section{Cell Viability Assay}

Different concentrations of Elotuzumab $(0,1,10,50,100$, $500,1000 \mathrm{ug} / \mathrm{mL}$ ) and PBMC were added to four cell types (UW, UR, UE, URE). The cells were processed with CCK-8 kits to detect cell proliferation after cells were collected and sorted with CD138 antibody based on the manufacturer's instruction.

\section{Profiling the Gene Expression}

Illumina gene expression microarray data was obtained as described previously.

\section{Real-Time RT-PCR to Detect the CCL20 Gene Expression}

To detect the expression of CCL20 mRNA in cell lines and the samples obtained from MM patient, qPCR was performed using Step One Plus Real Time PCR system. CCL20 Forward: ATGTGCTGTACCAAGAGTTTGC; CCL20

Reverse: CCAATTCCATTCCAGAAAAGCC.

\section{Enzyme-Linked Immunosorbent Assay to Detect CCL20 Protein Expression}

CCL20 (MIP 3alpha) (peprotech, USA) KIT was used for the detection of CCL20 protein expression in the plasma of MM patients based on the manufacturer's instruction.

\section{Bioinformatics Analysis}

$\mathrm{R}$ language was used to download the chip expression spectrum data of GSE16791, GSE31504, and GSE51317 for probe conversion, data standardization, and batch correction. The Ggpurb package was used to analyze the expression of CCL20.

\section{Xenograft Model}

Female NOD-SCID mice aged 4-5 weeks were purchased from Beijing Hua Fukang Bioscience Company (Beijing, China) and were housed and monitored in a pathogen-free environment. All animal studies were approved by the Research Ethics Committee of China Medical University with China Medical University Application for Laboratory Animal Welfare and Ethical review (201702 Edition). $1 \times 10^{7}$ U266/R10R cells were injected subcutaneously into NODSCID mice. The animal treatment was initiated after the detection of palpable tumors, approximately 12 days following the injection. Mice were randomly placed in two groups of three mice each using a computer-based randomization, 
with the control group (lenalidomide+NS) and the experimental group (lenalidomide+CCL20) to observe the effect of CCL20 reverse the resistance of lenalidomide. The drug was injected intraperitoneally. Control group: lenalidomide $25 \mathrm{mg} /$ $\mathrm{kg} / \mathrm{d}+\mathrm{NS}$; experimental group: lenalidomide $25 \mathrm{mg} / \mathrm{kg} / \mathrm{d}+$ CCL20 300ng $/ \mathrm{kg} / \mathrm{d}$. The drug was administered continuously for 7 days. Tumor growth was monitored every 3 days for about 3 weeks. Serial measurements of xenograft growth were performed, and tumor volume $(\mathrm{V})$ was calculated using the formula: $\mathrm{V}=0.5 \times \mathrm{a} \times \mathrm{b}^{2}$, where $\mathrm{a}$ and $\mathrm{b}$ are the longest and shortest tumor diameters, respectively. At the end of the study, the animals were killed and the tumors were collected.

\section{Statistical Analysis}

An independent sample $t$-test was employed to analyze any significant difference in the two samples using SPSS 23.0 software. The differences among multiple groups were analyzed using a One-way Analysis of Variance (ANOVA). Statistical significance in this study is established at the priori value of $p<0.05$ level of significance.

\section{Results}

\section{Generation of Elotuzumab and Lenalidomide Resistant Myeloma Cells}

Both the single drug resistance (U266/EloR, UE), and the dual drug resistance (U266/R10R/EloR, URE) were constructed using the continuous stimulation culture of Elotuzumab. Increasing the concentration of Elotuzumab resulted in the gradual decline in the viability of the sensitive cell lines (UW). However, there was no significant change in the cell viability of Elotuzumab resistant cell line (UE) (Figure 1A); the cell viability of the dual drug-resistant cell line (URE) has a lower cell viability compared with the lenalidomide-resistant cell line (UR) treated with different concentrations of Elotuzumab (Figure 1B). The results showed that the UE and URE cells have stable resistance to Elotuzumab.

\section{CCL20 Expression Decreased in Resistant Myeloma Cell}

The gene expression microarray was used in detecting the gene expression differences within the four myeloma cells (UW, UR, UE, and URE), to further explore the possible mechanism of resistance. The results showed that the expression of CCL20 was significantly down-regulated in drug-resistant cell lines (Figure 2A). RT-qPCR data further confirmed this result (Figure 2B). Also, there was a detection of the expression of CCL20 in the cells of MM patients. We collected 27 samples from patients with newly diagnosed MM (NDMM), and 5 samples from relapsed refractory MM (RRMM) patients with exposure to lenalidomide. The results showed that compared with NDMM, the expression of CCL20 mRNA in RRMM was lower $(p<0.01$; Figure 2C). Furthermore, the data from the GEO database showed that the expression of CCL20 in the bone marrow plasma cells of RRMM patients was lower than that of NDMM patients, especially the MM patients with exposure to lenalidomide, which was consistent with PCR results (Figure 2D). Finally, fresh plasma was collected from 5 NDMM patients and 5 RRMM patients exposed to lenalidomide. The average content of CCL20 was $24.44 \pm 0.88 \mathrm{pg} / \mathrm{mL}$ in the plasma of patients

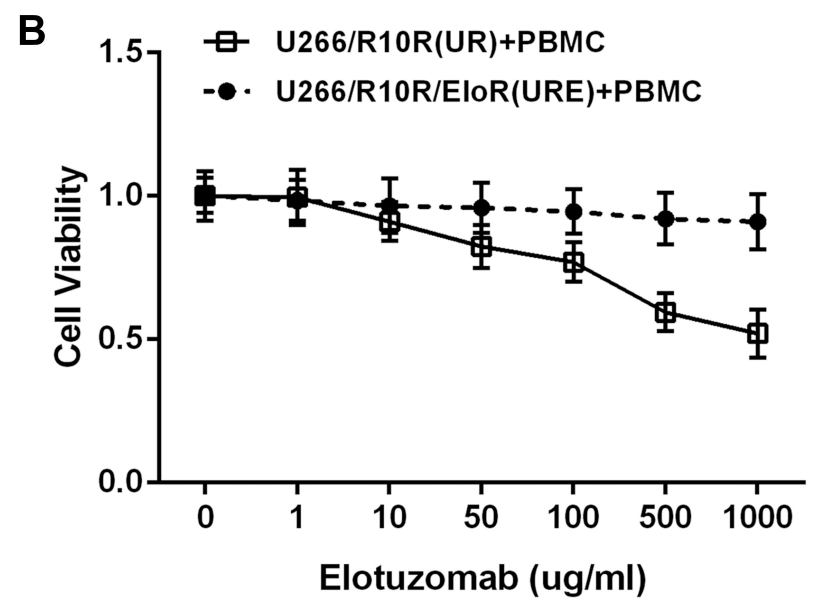

Figure I Cell viability of myeloma cells to Elotuzumab. (A) The cell viability of sensitive (U266/WT, UW) and Elotuzumab resistant (U266/EloR, UE) cell lines to Elotuzumab (ELO) coculture with peripheral blood mononuclear cells (PBMC). (B) The cell viability of lenalidomide resistance (U266/RIOR, UR) and double resistance to lenalidomide plus Elotuzumab (U266/RIOR/EloR, URE). 

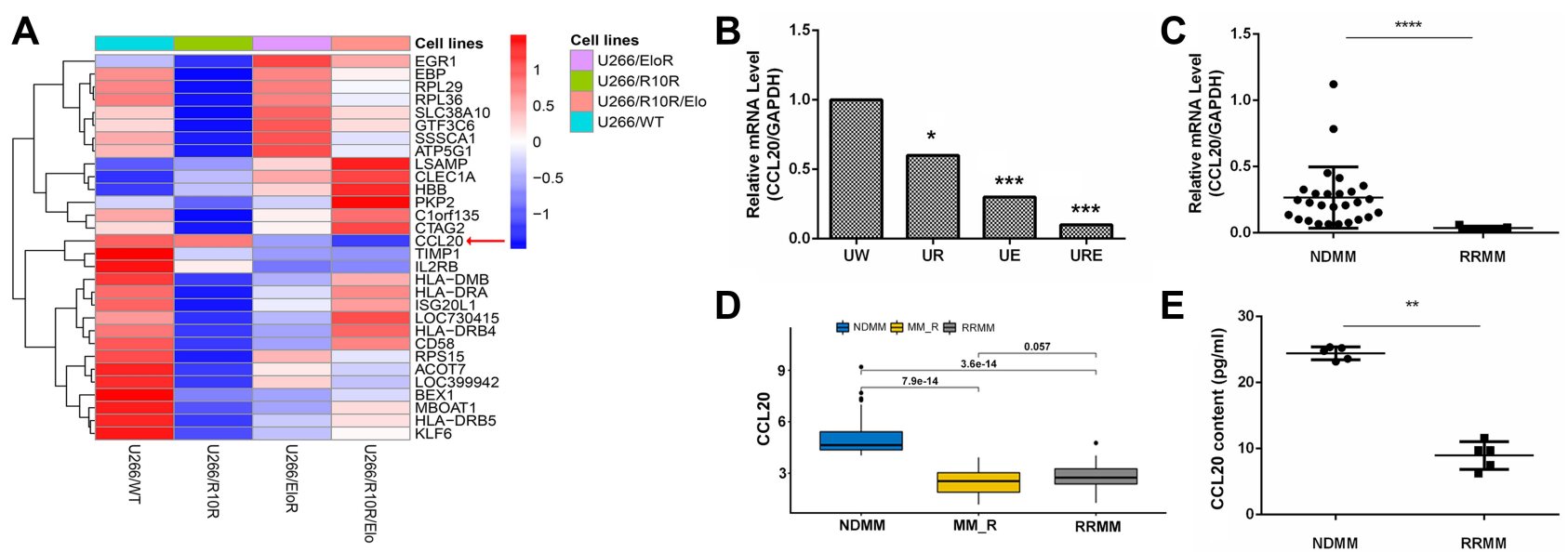

Figure 2 CCL20 expression decreased in resistant myeloma cell. (A) Heatmap for gene expression of four myeloma cells: UW, UR, UE, URE. (B) RT-qPCR to verify the relative expression of CCL20 gene in myeloma cell lines. (C) CCL20 gene expression in MM patient samples, 27 samples of bone marrow fluid from patients with newly diagnosed MM (NDMM) and 5 samples from relapsed refractory MM (RRMM) exposed to lenalidomide. (D) Bioinformatics analysis of CCL20 relative gene expression in NDMM and RRMM patient samples. MM_R is the patient who is resistant to lenalidomide. (E) CCL20 protein expression in plasma of NDMM and RRMM patients by ELISA. “*”. Indicates a $p$ value $<0.05$, “***'Indicates a $p$ value $<0.0$ I, “****”Indicates a $p$ value $<0.00$ I, “*****'Indicates a $p$ value $<0.000$ I.
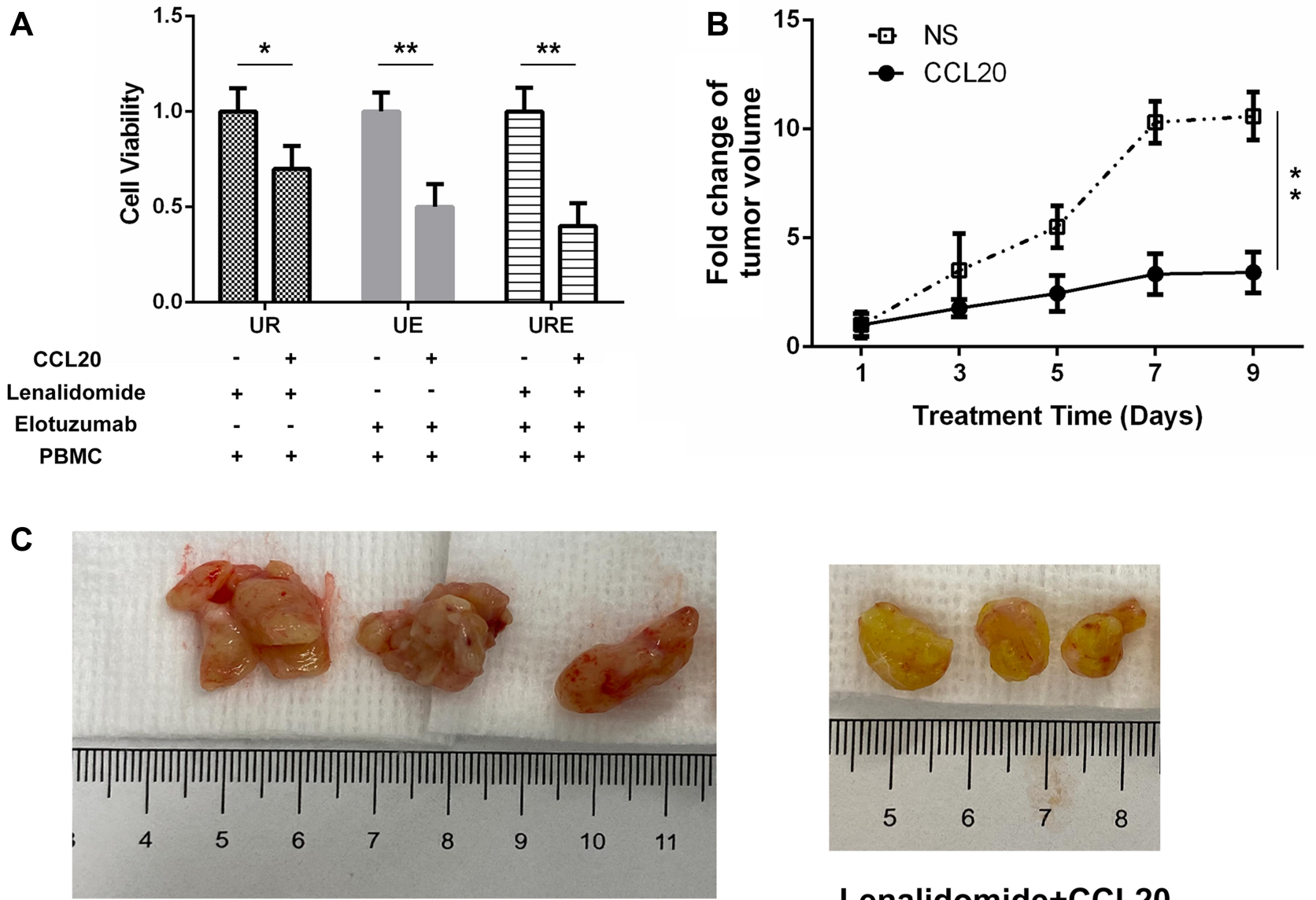

\section{Lenalidomide+NS}

\section{Lenalidomide+CCL20}

Figure 3 CCL20 increases drug sensitivity in vitro and in vivo. (A) Cell viability of UR, UE, URE cells treated with lenalidomide or Elotuzumab with or without CCL20. (B) Tumor volume growth rate of UR xenograft mice in the experimental group (lenalidomide+CCL20) and control group (lenalidomide+NS). (C) Tumor volume (blank control group on the left, experimental group on the right). “*”'Indicates a $p$ value $<0.05$, “***”Indicates a $p$ value $<0.0$ I. 
with NDMM and $8.97 \pm 1.88 \mathrm{pg} / \mathrm{mL}$ in RRMM $(p<0.01)$ (Figure 2E). These data indicated a decline in the expression of CCL20 in resistant myeloma cells.

\section{CCL20 Increases the Sensitivity of Drug-Resistant Myeloma Cells to Immunomodulatory Drugs}

The experimental units were divided into three groups: lenalidomide, Elotuzumab, and lenalidomide +Elotuzumab, with the addition of PBMC to all treatment groups. The results showed that in each resistant cell group (UR, UE, URE), the cell viability with the addition of CCL20 were significantly lower compared with that without CCL20 (Figure 3A), this could be an indication of the ability of CCL20 in partially restoring the drug sensitivity of drug-resistant myeloma cells. While adding chemokine CCL20 without lenalidomide or Elotuzumab did not significantly change the proliferation of drug-resistant cells (Supplement Figure 1).

\section{CCL20 Increases the Sensitivity of Resistant Myeloma Mice to Lenalidomide in vivo}

Drug resistance U266/R10R cells xenograft NOD-SCID mice was constructed by subcutaneous injection to verify the effect of CCL20 on lenalidomide resistance in vivo. With the prolongation of the lenalidomide treatment, the growth rate of the tumor volume in the experimental group (lenalidomide + CCL20) was significantly slower compared with the control group (lenalidomide + NS) (Figure 3B). The tumor volume in the experimental group was significantly smaller than that of the control group (Figure 3C).

\section{Discussion}

Despite the rapid advancements in myeloma immunotherapy in recent years, the mechanism of immunotherapy resistance has been a major cause of concern to researchers. These outcomes likely reflect the complex and highly regulated nature of the immune system. The continuous ability of immunotherapy to play its fundamental role and the prevention of resistance to immunotherapy are important topics in the treatment of myeloma. The vital immunotherapeutic agents in myeloma are immunomodulators and monoclonal antibodies. Lenalidomide is a widely used immunomodulator, which inhibits the secretion of inflammatory agents, by directly inducing the phosphorylation of CD28 on T cells, leading to the activation of the downstream target factors, then activating $\mathrm{T}$ cells and the enhancement of various mechanisms mediated by the NK cells to achieve antitumor effects. ${ }^{6,7}$ Elotuzumab targets and labels the CS1 protein on the surface of multiple myeloma cells, and inhibits the adhesion between the myeloma cells and the stromal cells of the bone marrow, regulates the microenvironment of the MM bone marrow, and reduces the growth-stimulating factors on myeloma cells. ${ }^{8-11}$ The combination of immune agents Elotuzumab and Rd (lenalidomide plus dexamethasone) has obvious advantages compared with $\mathrm{Rd}^{12}$ However, some patients are still not sensitive to the E-Rd regimen. The mechanism of resistance to Elotuzumab and lenalidomide is not clear yet.

To further improve the efficacy of immunotherapy and clarify the mechanism of immunotherapeutic resistance, the myeloma cell model of resistance to Elotuzumab, and dural resistance to both Elotuzumab and lenalidomide were initially constructed. From the gene expression profile, we focused on the different expression of genes affecting the functions of the immune cells. We subsequently discovered a decrease in the expression of CCL20 (chemokine (C-C motif) ligand 20 and macrophage infectious protein-3 $\alpha$, mip- $3 \alpha$ ) in the drugresistant cells. We also verified the down-regulation of CCL20 involved in the development of drug resistance in the immunotherapy of myeloma cells both in vivo and in vitro.

CCL20 is a member of the chemokines family which are small chemoattractant proteins involved in the regulation of leukocyte migration, adhesion, and proliferation. Chemokines are classified into four families $(\mathrm{C}, \mathrm{CC}, \mathrm{CXC}$, and $\mathrm{CX} 3 \mathrm{C}$ ) based on the number and spacing of cysteine residues and exert their biological effects by interacting with specific cell surface receptors that belong to transmembrane G protein-coupled receptor family. CCL20 and its selective receptor CC-chemokine receptor 6 (CCR6), ${ }^{13,14}$ known to be responsible for the chemoattraction of dendritic, $\mathrm{T}$, and $\mathrm{B}$ cells, have been recently involved in rheumatoid arthritis synovitis ${ }^{13}$ and $\mathrm{OC}$ activation. ${ }^{20}$ The expression of CCL20 is regulated by numerous cytokines such as TNF- $\alpha$, IL-17, and IFN- $\gamma$, causing the chemotaxis of inflammatory cells, and affecting the proliferation and metastasis of tumor cells. ${ }^{15,16}$ Numerous studies have shown that CCL20 has an abnormal expression in a variety of cancers such as esophageal cancer, lung cancer, and colorectal cancer. The increased expression of CCL20 after paclitaxel chemotherapy in 
triple-negative breast cancer activated the NF- $\mathrm{B}$ pathway, promoted the self-renewal of breast cancer stem cells/stem like cells, and participated in the occurrence of drug resistance. $^{17-19}$ These data suggested the resistance participation of CCL20 in some forms of cancer.

Perusing through existing literature indicated that only a few studies have investigated CCL20 in the treatment of myeloma. Giuliani et al found that myeloma cell lines U266, JJN3, and the EBV-positive cell line ARH-77 express CCL20 mRNA by PCR. The presence of CCR6 at mRNA level was found in U266, OPM2, and ARH-77. CCL20 mRNA expression was also investigated in 4/22 purified CD138+ MM cells. ${ }^{20}$ In the present study, we used U266 cells with higher expressions of CCL20 to construct the resistance model. From the patient sample and bioanalysis, there were evidences to infer that CCL20 has different expressions in sensitive and resistant cells, and this partly suggested that CCL20 could be one of the mechanisms of immunotherapy resistance. Also, it has been recently established that CCL20 is related to the regulation of bone marrow microenvironment in myeloma cells and participates in the occurrence of myeloma osteopathy. $^{21,22}$ Besides, increment in CCL20 can promote T-cell-mediated antitumor immunity through CCL20-CCR6dependent dendritic cell regulation, thus providing a new target for immunotherapy. ${ }^{23}$ We discovered that increasing CCL20 can enhance the anti-myeloma effects of lenalidomide. We speculated that the mechanism may be attributed to increasing lymphocyte chemotaxis to the tumor areas and assisting in cellmediated immunity. In RRMM, the down-regulation of CCL20 resulted in a decreasing immunotherapeutic effect.

Progressive attention has been given to the role of CCL20-CCR6 axis in immune regulation and mechanism in autoimmune diseases. Our study preliminarily explored the role of CCL20-CCR6 axis in the resistance of myeloma immunotherapy with the aim of providing a new target for immunotherapy, reverse the resistance of myeloma cells to immunotherapy. To improve the efficacy and safety of MM treatments, there is need for further studies on the downstream effector cells and mechanism.

\section{Conclusions}

We found that the expression of CCL20 was decreased in lenalidomide and Elotuzumab resistant U266 cells and RRMM patients. CCL20 could possibly increase the sensitivity of lenalidomide in vitro and in vivo. Our study preliminarily found the role of CCL20-CCR6 axis in the resistance of myeloma immunotherapy and may provide a new target for immunotherapy, and reverse the resistance of myeloma cells.

\section{Consent for Publication}

Not applicable.

\section{Acknowledgments}

Myeloma drug-naive U266 cell line (U266/WT, UW) and Lenalidomide resistant cell line (U266/R10R, UR) were provided by Professor Robert Orlowski in the MD Anderson Cancer Centre. Also, the Elotuzumab and lenalidomide were provided by Dr. Robert Orlowski. The microarray data was provided by Dr. Robert Orlowski.

\section{Author Contributions}

All authors made a significant contribution to the work reported, whether that is in the conception, study design, execution, acquisition of data, analysis and interpretation, or in all these areas; took part in drafting, revising or critically reviewing the article; gave final approval of the version to be published; have agreed on the journal to which the article has been submitted; and agree to be accountable for all aspects of the work.

\section{Funding}

This study was supported by the Natural Science Foundation of Liaoning Province (201800875, 20180551257). The funder Aijun Liao designed the study and the funder Huihan Wang performed the experiments and wrote the manuscript.

\section{Disclosure}

The authors declare that they have no competing interests.

\section{References}

1. Rajkumar SV. Treatment of multiple myeloma. Nat Rev Clin Oncol. 2011;8(8):479-491. doi:10.1038/nrclinonc.2011.63

2. Al-Hujaily EM, Oldham RA, Hari P, Medin JA. Development of novel immunotherapies for multiple myeloma. Int J Mol Sci. 2016;17(9). doi:10.3390/ijms 17091506

3. Einsele H, Schreder M. Treatment of multiple myeloma with the immunostimulatory SLAMF7 antibody elotuzumab. Ther Adv Hematol. 2016;7(5):288-301. doi:10.1177/2040620716657993

4. Lonial S, Kaufman J, Reece D, Mateos MV, Laubach J, Richardson P. Update on elotuzumab, a novel anti-SLAMF7 monoclonal antibody for the treatment of multiple myeloma. Expert Opin Biol Ther. 2016;16 (10):1291-1301. doi:10.1080/14712598.2016.1221920

5. Magen H, Muchtar E. Elotuzumab: the first approved monoclonal antibody for multiple myeloma treatment. Ther Adv Hematol. 2016;7 (4):187-195. doi:10.1177/2040620716652862

6. Zhu YX, Kortuem KM, Stewart AK. Molecular mechanism of action of immune-modulatory drugs thalidomide, lenalidomide and pomalidomide in multiple myeloma. Leuk Lymphoma. 2013;54(4):683-687. doi: $10.3109 / 10428194.2012 .728597$ 
7. Fink EC, Ebert BL. The novel mechanism of lenalidomide activity. Blood. 2015;126(21):2366-2369. doi:10.1182/blood-2015-07-567958

8. Grosicki S, Barchnicka A. Elotuzumab: a novel immune-stimulating therapy to treat multiple myeloma. Expert Rev Hematol. 2016;9 (7):621-628. doi:10.1080/17474086.2016.1199947

9. Balasa B, Yun R, Belmar NA, et al. Elotuzumab enhances natural killer cell activation and myeloma cell killing through interleukin-2 and TNF- $\alpha$ pathways. Cancer Immunol Immunother. 2015;64 (1):61-73. doi:10.1007/s00262-014-1610-3

10. Collins SM, Bakan CE, Swartzel GD, et al. Elotuzumab directly enhances NK cell cytotoxicity against myeloma via CS1 ligation: evidence for augmented NK cell function complementing ADCC. Cancer Immunol Immunother. 2013;62(12):1841-1849. doi:10.1007/ s00262-013-1493-8

11. Veillette A, Guo H. CS1, a SLAM family receptor involved in immune regulation, is a therapeutic target in multiple myeloma. Crit Rev Oncol Hematol. 2013;88(1):168-177. doi:10.1016/j. critrevonc.2013.04.003

12. Lonial S, Dimopoulos M, Palumbo A, et al. Elotuzumab therapy for relapsed or refractory multiple myeloma. $N$ Engl J Med. 2015;373 (7):621-631. doi:10.1056/NEJMoa1505654

13. Comerford I, Bunting M, Fenix K, et al. An immune paradox: how can the same chemokine axis regulate both immune tolerance and activation?: CCR6/CCL20: a chemokine axis balancing immunological tolerance and inflammation in autoimmune disease. Bioessays. 2010;32(12):1067-1076. doi:10.1002/bies.201000063

14. Lee AY, Phan TK, Hulett MD, Körner H. The relationship between CCR6 and its binding partners: does the CCR6-CCL20 axis have to be extended? Cytokine. 2015;72(1):97-101. doi:10.1016/j. cyto.2014.11.029

15. Frick VO, Rubie C, Keilholz U, Ghadjar P. Chemokine/chemokine receptor pair CCL20/CCR6 in human colorectal malignancy: an overview. World J Gastroenterol. 2016;22(2):833-841. doi:10.3748/ wjg.v22.i2.833
16. Lee AY, Körner H. CCR6 and CCL20: emerging players in the pathogenesis of rheumatoid arthritis. Immunol Cell Biol. 2014;92 (4):354-358. doi:10.1038/icb.2013.97

17. Chen W, Qin Y, Wang D, et al. CCL20 triggered by chemotherapy hinders the therapeutic efficacy of breast cancer. PLoS Biol. 2018;16 (7):e2005869. doi:10.1371/journal.pbio.2005869

18. Wei W, Zhao X, Zhu J, et al. IncRNA-u50535 promotes the progression of lung cancer by activating CCL20/ERK signaling. Oncol Rep. 2019;42(5):1946-1956. doi:10.3892/or.2019.7302

19. Wang D, Yuan W, Wang Y, et al. Serum CCL20 combined with IL-17A as early diagnostic and prognostic biomarkers for human colorectal cancer. J Transl Med. 2019;17(1):253. doi:10.1186/ s12967-019-2008-y

20. Mo"ller C, Stro“mberg T, Juremalm M, Nilsson K, Nilsson G. Expression and function of chemokine receptors in human multiple myeloma. Leukemia. 2003;17:203-210. doi:10.1038/sj.leu.2402717

21. Palma BD, Guasco D, Pedrazzoni M, et al. Osteolytic lesions, cytogenetic features and bone marrow levels of cytokines and chemokines in multiple myeloma patients: role of chemokine (C-C motif) ligand 20. Leukemia. 2016;30(2):409-416. doi:10.1038/leu.2015.259

22. Giuliani N, Lisignoli G, Colla S, et al. CC-chemokine ligand 20/ macrophage inflammatory protein-3 $\alpha$ and $\mathrm{CC}$-chemokine receptor 6 are overexpressed in myeloma microenvironment related to osteolytic bone lesions. Cancer Res. 2008;68(16):6840-6850. doi:10.1158/ 0008-5472.CAN-08-0402

23. Lu Y, Yi Q. Utilizing $\mathrm{T}(\mathrm{H}) 9$ cells as a novel therapeutic strategy for malignancies. Oncoimmunology. 2013;2(3):e23084. doi:10.4161/ onci.23084
OncoTargets and Therapy

\section{Publish your work in this journal}

OncoTargets and Therapy is an international, peer-reviewed, open access journal focusing on the pathological basis of all cancers, potential targets for therapy and treatment protocols employed to improve the management of cancer patients. The journal also focuses on the impact of management programs and new therapeutic

Submit your manuscript here: https://www.dovepress.com/oncotargets-and-therapy-journ

\section{Dovepress}

agents and protocols on patient perspectives such as quality of life, adherence and satisfaction. The manuscript management system is completely online and includes a very quick and fair peer-review system, which is all easy to use. Visit http://www.dovepress.com/ testimonials.php to read real quotes from published authors. 